\title{
Modelo para redistribuição de cotas de emissão de gases do efeito estufa baseado em medidas de eficiência técnica
}

\author{
Greenhouse gases emission quotas distribution \\ model based on technical efficiency measurements
}

\author{
Marco Aurélio Macedo' \\ João Carlos Correia Baptista Soares de Mello² \\ Eliane Gonçalves Gomes ${ }^{3}$
}

\begin{abstract}
Resumo: O Protocolo de Kyoto representa um importante compromisso ecológico assumido por diversos países, com destaque à questão das cotas de carbono permitidas a cada país emitir. Para decidir de maneira mais justa sobre esta divisão, recorreu-se aos modelos Data Envelopment Analysis - DEA. É aqui proposta uma variante do modelo DEA com Ganhos de Soma Zero (DEA-GSZ), que permite o comportamento híbrido dos retornos de escala na fronteira de eficiência. Este modelo foi usado no estudo da redistribuição de cotas de $\mathrm{CO}_{2}$ entre países. Os resultados mostram-se mais adequados do que aqueles obtidos com o modelo DEA-GSZ clássico.
\end{abstract}

Palavras-chave: DEA. Ganhos de soma zero. Retornos híbridos de escala. Emissões de $\mathrm{CO}_{2}$.

\begin{abstract}
The Kyoto Protocol is an important ecological compromise undertaken by many countries. One of its most important points is the carbon emission quota allowed to each country. In order to fairly allocate these quotas, we used the DEA models. The Zero Sum Gains Data Envelopment Analysis - DEA (ZSG-DEA) model with hybrid returns to scale was proposed to solve this problem. This model was used to reallocate the $\mathrm{CO}_{2}$ quotas among the countries. The results found were more adequate than those obtained by the classic ZSG-DEA models.
\end{abstract}

Keywords: DEA. Zero sum gains. Hybrid returns to scale. $\mathrm{CO}_{2}$ emissions.

\section{Introdução}

A intensificação do efeito estufa é a principal causa do aquecimento global e consequência direta do aumento das emissões de gases poluentes. Esse aumento é provocado principalmente por atividades antrópicas, indústrias, veículos em circulação e sistemas domésticos de aquecimento, além de atividades agropastoris, lixões e aterros sanitários.

O Protocolo de Kyoto é um instrumento que tem como objetivo fazer com que os países industrializados (chamados "países do Anexo I") reduzam, e controlem, as suas emissões combinadas de gases de efeito estufa em pelo menos 5\% em relação aos níveis de 1990, até o período entre 2008 e 2012. O elemento chave a ser controlado é o nível de emissão global. As emissões particulares dos países podem ser flexibilizadas, condicionadas a um balanço global rígido.
Os países signatários poderão reduzir as suas emissões em nível doméstico e/ou terão a possibilidade de aproveitar os chamados "mecanismos flexíveis" para cumprir seus compromissos: Implementação Conjunta, Comércio de Emissões e Mecanismo de Desenvolvimento Limpo. Estes mecanismos podem resultar em acordos entre os países poluentes. Poderá ser possível não só estabilizar o total produzido de poluição, mas também fazer com que as cotas admissíveis de poluição sejam alocadas entre os países da maneira mais eficiente. Outro aspecto importante na divisão de cotas de poluição é não permitir que países muito eficientes tecnologicamente venham a concentrar altas cotas de gases do efeito estufa em pequenas extensões territoriais. Isto causaria estragos severos e pontuais na camada de ozônio.

\footnotetext{
${ }^{1}$ Mestrado em Engenharia de Produção, Departamento de Engenharia de Produção, Universidade Federal Fluminense, Rua Passo da Pátria 156, São Domingos, CEP 24210-240, Niteroi - RJ, Brasil, E-mail: marconauff@ yahoo.com.br

${ }^{2}$ Departamento de Engenharia de Produção - Universidade Federal Fluminense, Rua Passo da Pátria 156, São Domingos, CEP 24210-240, Niteroi - RJ, Brasil, E-mail: jcsmello@ producao.uff.br

${ }^{3}$ Empresa Brasileira de Pesquisa Agropecuária (Embrapa) - SGE, Parque Estação Biológica, Av. W3 Norte Final, Asa Norte, CEP 70770-901, Brasília - DF, Brasil, E-mail: eliane.gomes@embrapa.br
}

Recebido em 6/2/2008 — Aceito em 1/7/2010

Suporte financeiro: CNPq. 
A alocação eficiente dos gases do efeito estufa entre os países poluidores pode ser estudada com modelos DEA. Como os gases do efeito estufa são o produto indesejável de um processo produtivo, eles podem ser modelados em DEA como outputs indesejáveis. Sobre o tema ver, entre outros, Hailu e Veeman (2001), Scheel (2001), Seiford e Zhu (2002), Färe e Grosskopf (2004), Jahanshahloo et al. (2005), Kuosmanen (2005), Vencheh, Kazemi Matin e Tavassoli Kajani (2005), Amirteimoori, Kordrostami e Sarparast (2006), Gomes e Lins (2008). Sobre previsão de emissões de gases do efeito estufa ver, por exemplo, Rodrigues et al. (2009).

A maioria dos modelos DEA presentes na literatura considera que os outputs indesejáveis podem ser reduzidos de forma independente, sem integração nem cooperação entre as unidades produtivas. Entretanto, nos casos de análise de eficiência com a presença de outputs indesejáveis, modelos DEA com Ganhos de Soma Zero (DEA-GSZ) (GOMES, 2003; LINS et al., 2003; GOMES; SOARES DE MELLO; LINS, 2003, 2004, 2005; GOMES et al., 2007; GOMES; LINS, 2008) podem ser úteis na determinação de diretrizes para as unidades em avaliação, já que a soma das quantidades produzidas por todas as DMUs pode ser vista como o limite máximo permitido e, assim, não deve ser ultrapassado. Isto significa que qualquer DMU que busque a fronteira pelo aumento da quantidade desse output deverá impor a perda dessa quantidade pelas demais unidades produtivas (para que a soma líquida seja zero). Isto provoca a redistribuição dessas quantidades pelas unidades em avaliação, com base nas medidas de eficiência. Um exemplo da aplicação destes modelos ao caso ambiental pode ser visto em Gomes e Lins (2008). Deve-se ressaltar que existem outros modelos que fazem redistribuição de inputs e/ou outputs em DEA: o modelo de alocação centralizada (LOZANO; VILLA, 2004), o algoritmo de alocação de recursos discretos (SOARES DE MELLO et al., 2006) e os modelos de fronteira esférica e elipsoidal (AVELLAR; MILIONI; RABELLO, 2007; AVELLAR, 2010), por exemplo. O primeiro assume como hipótese o modelo DEA BCC e que a determinação de alvos não é uma decisão individual de cada unidade, mas sim de um poder centralizado. O segundo, como o nome indica, serve apenas quando os recursos não possuem medida contínua. O último impõe uma forma funcional de fronteira, adaptada a modelos CCR.

No caso da emissão de gases poluentes, por exemplo, o modelo DEA-GSZ teria inserção no "mercado de carbono", cujo objetivo é aliviar o impacto na economia das partes do Anexo I e tornar viáveis os objetivos estabelecidos pelo Protocolo. No caso do Comércio de Emissões, há a previsão de que, como alguns países têm emissões abaixo do limite estabelecido pelo Protocolo de Kyoto (países não pertencentes ao Anexo I), haja a possibilidade de essa diferença ser coberta por um aumento na emissão de carbono pelos países do Anexo I. Ao assumir os resultados da abordagem DEA-GSZ, haveria uma negociação de cotas de emissão de carbono entre os dois grupos, sem alterar a emissão global. Já no Mecanismo de Desenvolvimento Limpo, os países emissores poderiam patrocinar projetos no outro grupo para reduzir ainda mais as emissões desse grupo. Com isso, aumentar-se-iam suas emissões, de modo a não alterar a emissão global.

Nesse contexto, este artigo propõe uma abordagem para modelagem desses outputs indesejáveis, baseada na proposta de Gomes (2003) e Gomes e Lins (2008). O modelo aqui usado objetivou obter cotas individuais diferenciadas, segundo as dimensões demográfica, geográfica e econômica dos países. Especificamente, foi usado o modelo DEA-GSZ com retornos híbridos de escala para a proposição de um possível quadro sobre as trocas de cotas de carbono ou a realocação das emissões globais.

\section{Modelos de Análise de Envoltória de Dados com Ganhos de Soma Zero}

\subsection{Conceitos gerais}

Os modelos DEA clássicos, tanto o modelo CCR (CHARNES; COOPER; RHODES, 1978) quanto o modelo BCC (BANKER; CHARNES; COOPER, 1984), além de todas as suas variantes, supõem total liberdade de produção, ou seja, a produção de uma DMU não interfere na produção das demais. A mesma suposição pode ser feita para a utilização de recursos. Entretanto, em alguns casos essa liberdade não existe. No caso de competições, por exemplo, se for considerado como output uma variável relacionada aos resultados obtidos (LINS et al., 2003; VILLA, LOZANO, 2004; GOMES, AVELLAR, 2005), a melhora de posição de qualquer competidor implica na perda de posição de um ou mais de seus adversários.

Nos modelos chamados de DEA com Ganhos de Soma Zero (DEA-GSZ) (GOMES, 2003; LINS et al., 2003; GOMES; SOARES DE MELLO; LINS, 2003, 2004, 2005; GOMES et al., 2007; GOMES, LINS, 2008), uma DMU ineficiente que busque a fronteira pela redução de inputs (ou aumento de outputs) imputará às demais o aumento do valor de seus inputs (ou redução do valor de seus outputs) de modo a manter a soma total constante. Assim, os modelos DEA-GSZ têm aplicação direta nos estudos de alocação ou (re)alocação de recursos (ou de produção), em que a restrição de soma constante seja uma necessidade da modelagem.

Nos modelos DEA-GSZ há a restrição adicional aos modelos DEA clássicos de que a soma líquida dos 
ganhos deve ser zero. E, ao contrário do que acontece tradicionalmente, o modo como uma DMU atinge seu alvo na fronteira pode implicar na alteração da forma da fronteira eficiente.

Há várias formas ou estratégias para uma DMU ineficiente buscar seu alvo dentro dessas condições. Em Gomes (2003), são propostas estratégias para a busca de alvos, destacando-se a estratégia de redução proporcional. Nessa estratégia, a DMU que busca eficiência (busca a fronteira) precisa perder determinadas unidades de input (ou ganhar output). Para que a soma seja mantida constante, o ganho (ou perda, no caso de outputs) das outras DMUs deve ser proporcional aos seus níveis de input (output). Isto significa que quem tem menor nível de input (output) ganha (perde) menos, quem tem maior nível de input (output) ganha (perde) mais.

Há também a possibilidade de mais de uma DMU procurar maximizar a eficiência, o que pode ser feito em competição ou cooperação. $O$ caso mais interessante em modelagem GSZ é aquele no qual DMUs formam um grupo de cooperação. No paradigma do DEA-GSZ, a busca em cooperação significa que as DMUs deste grupo tentam alocar determinada quantidade de input (ou retirar quantidade de output) apenas às DMUs não pertencentes ao grupo.

No caso geral, de múltiplas DMUs atuarem em regime de cooperação, o modelo DEA-GSZ é um Problema de Programação Não Linear Multiobjetivo (GOMES, 2003). Problemas deste tipo conduzem frequentemente ao uso de meta-heurísticas, devido à quantidade de variáveis e DMUs. No entanto, para a estratégia de redução proporcional, em Gomes (2003) prova-se que o modelo é reduzido a um modelo de Programação Não Linear Mono-objetivo, segundo o Teorema da Proporcionalidade das Eficiências em Estratégia Proporcional. Seu enunciado estabelece que, no problema de várias DMUs em cooperação na busca de alvos com estratégia proporcional, as eficiências das DMUs no modelo DEA-GSZ são diretamente proporcionais às suas eficiências no modelo DEA clássico.

No caso em que todas as DMUs ineficientes formam um único grupo de cooperação e buscam eficiência na fronteira de eficiência DEA clássica, a aplicação do modelo DEA-GSZ fará com que ocorra a redistribuição total da variável de soma constante. Após essa redistribuição, todas as DMUs pertencerão à fronteira eficiente, ou seja, todas serão $100 \%$ eficientes.

Esta nova fronteira DEA, aqui chamada de fronteira DEA uniformizada ou de máxima eficiência, estará localizada em níveis inferiores aos da fronteira DEA do modelo clássico, já que as DMUs eficientes ganham unidades de input (ou perdem unidades de output) para compensar a perda (ou ganho) das unidades ineficientes, de modo a manter a soma constante. Esta situação de máxima eficiência pode ser vista como "ideal" por órgãos reguladores, já que será apresentada ao decisor a distribuição de recursos (ou produtos) que faz com que todas as unidades sejam $100 \%$ eficientes.

Para a construção da fronteira uniformizada de forma direta, em que as DMUs ineficientes formam um único grupo de cooperação $W$, Gomes (2003) provou o Teorema da Determinação do Alvo, o qual estabelece que

[...] o alvo da DMU em análise no modelo DEA-GSZ de estratégia proporcional é igual ao alvo no caso clássico multiplicado pelo coeficiente de redução [...] Gomes (2003, p. 28).

Este teorema, juntamente com o Teorema da Proporcionalidade das Eficiências em Estratégia Proporcional, permite reduzir a solução do Problema de Programação Não Linear a uma única Equação.

Assim, para os modelos CCR e BCC, com orientação a inputs, tem-se a Equação (1), na qual $h_{R i}$ e $h_{i}$ são, respectivamente, as eficiências nos modelos DEA-GSZ e DEA clássico; $W$ é o grupo de DMUs em cooperação; $r_{i j}=h_{i-I} / h_{j-I}$ é o fator de proporcionalidade resultante do emprego da estratégia proporcional, na orientação a inputs. Já a Equação (2) é válida para modelos orientados a outputs, e $q_{i j}=h_{i-O} / h_{j-O}$ é o fator de proporcionalidade.

$$
\begin{gathered}
h_{R i}=h_{i}\left(1+\frac{\sum_{j \in W}\left[x_{j}\left(1-r_{i j} h_{R i}\right)\right]}{\sum_{j \notin W} x_{j}}\right) \\
h_{R i}=h_{i}\left(1-\frac{\sum_{j \in W}\left[y_{j}\left(q_{i j} h_{R i}-1\right)\right]}{\sum_{j \notin W} y_{j}}\right)
\end{gathered}
$$

Cabe ressaltar que na literatura há referência a outros modelos que usam a restrição de soma constante em DEA. Destacam-se os trabalhos de Avellar, Milioni e Rabello (2007, 2005), Avellar (2010) e Villa e Lozano (2004). Os primeiros propõem modelos tipo DEA CCR baseados em inputs/outputs limitados, nos quais a distribuição dos recursos/produtos pode ser influenciada tanto pelos inputs como pelos outputs envolvidos. A construção desses modelos tomou como base o perfil geométrico da fronteira CCR tridimensional, que é substituída por uma fronteira hiperbólica, esférica ou elipsoidal, em função da natureza da variável a distribuir. Repare-se que estes modelos postulam um tipo de função para a fronteira, ao contrário do DEA clássico. Guedes (2007) chama a este tipo de modelo de "DEA paramétrico". Já Villa e Lozano (2004) propõem um modelo DEA BCC em duas fases, chamado de Constant Sum of Outputs (CSO). Na primeira fase deste modelo, resolve-se o modelo DEA BCC clássico; na segunda, calculam-se a contração radial das saídas, os alvos de soma constante e os de soma não constante. 


\subsection{Modelos de Análise de Envoltória de Dados com Ganhos de Soma Zero e Retornos Híbridos de Escala}

Modelos DEA híbridos são assim chamados por apresentarem uma configuração em que o modelo, em relação aos retornos de escala, é tratado de maneira distinta, híbrida, em diferentes partes da fronteira de eficiência. Estes modelos possuem duas regiões de operação: retornos crescentes - retornos constantes (IRS) ou retornos constantes - retornos decrescentes (DRS). No modelo híbrido IRS deseja-se dar ganhos crescentes de escala a pequenos valores de entrada e ganhos proporcionais a grandes valores. Já no modelo DRS dão-se ganhos constantes de escala para pequenos valores e ganhos decrescentes para grandes valores.

Do ponto de vista da formulação matemática, estes são casos particulares dos modelos DEA clássicos CCR e BCC. A diferença entre os modelos está na restrição de convexidade, inexistente no modelo CCR e igual a $\sum_{j} \lambda_{j}=1$ no modelo BCC. No modelo DEA híbrido com retornos crescentes de escala esta restrição é escrita como $\sum_{j} \lambda_{j} \geq 1$, e $\sum_{j} \lambda_{j} \leq 1$ para o modelo com retornos decrescentes (COOPER et al., 2000).

Como os modelos híbridos IRS e DRS são casos particulares dos modelos DEA clássicos, estes também serão casos particulares dos modelos DEA-GSZ CCR e BCC (MACEDO, 2005). Dessa forma, os teoremas provados para os casos CCR e BCC continuam válidos para o caso híbrido. Como consequência, as Equações (1) e (2) são válidas para o caso DEA-GSZ com retornos híbridos de escala, com as medidas de eficiência calculadas segundo os modelos escolhidos.

\subsection{Acréscimo de variáveis em modelos de Análise de Envoltória de Dados com Ganhos de Soma Zero}

A escolha de variáveis em modelos DEA deve ser bastante criteriosa, já que determina o que o modelo irá medir. É importante evitar a inclusão de variáveis pouco relevantes, já que estas podem fazer com que DMUs não eficientes atinjam a fronteira de eficiência por conta dessa variável. Em modelos DEA clássicos, a inclusão de variáveis não reduz a eficiência de qualquer DMU, mas pode aumentar o valor de algumas.

Em modelos DEA-GSZ, deve-se ter o mesmo cuidado na escolha das variáveis. Assim como em DEA clássico, a inclusão de variáveis ao modelo DEA-GSZ não reduzirá a eficiência de DMU alguma. No entanto, pode diminuir a parcela de recursos a serem realocados a determinada DMU. Tal fato ocorre porque o modelo DEA-GSZ é análogo a um jogo de soma zero. Assim, se uma DMU melhora a sua eficiência com a variável acrescentada, obterá mais recursos, o que significa que outras perderão recursos.

\section{Redistribuição das emissões de $\mathrm{CO}_{2}$}

\subsection{Modelagem}

Quando as regras do Protocolo de Kyoto foram criadas não foram considerados os diversos fatores que compõem o cenário socioeconômico de cada país. Apenas determinou-se um percentual único para que os países do Anexo I estimassem sua redução. Os demais países, apesar de assinarem e/ou ratificarem o Protocolo, não têm obrigatoriedade de redução de emissões.

As emissões de $\mathrm{CO}_{2}$ geradas pelos países são resultado do progresso e advindas das atividades sociais, industriais e econômicas neles desenvolvidas. A esse "malefício" gerado é agregado um determinado "benefício" produzido. Esta medida entre a quantidade de bônus e a quantidade de ônus realizada por cada país é informação fundamental para uma proposta de divisão de cotas de $\mathrm{CO}_{2}$ mais "justa". O objetivo é permitir maiores emissões aos países mais eficientes, isto é, que melhor aproveitam essas emissões.

Ramanathan $(2002,2005,2006)$ usa DEA para estudar várias relações entre PIB, energia e emissões de $\mathrm{CO}_{2}$. Gomes e Lins (2008) propuseram o uso do modelo DEA-GSZ CCR para tratar o problema da redistribuição das emissões de $\mathrm{CO}_{2}$ entre 64 países do mundo. Gomes (2003) apresenta os resultados da modelagem DEA-GSZ BCC deste mesmo problema. Estes autores usaram como variáveis população, consumo de energia, PIB (como outputs) e emissões de $\mathrm{CO}_{2}$ como output indesejável. Como há diversas formas de modelar outputs indesejáveis (SCHEEL, 2001), os autores modelaram-no como input, já que era desejado que sua quantidade fosse minimizada. Estas mesmas variáveis foram usadas por Avellar (2010) para a redistribuição das emissões (input de soma constante), com uso do modelo de fronteira elipsoidal.

As variáveis usadas nesse modelo podem ser criticadas por dois tipos de problemas: redundância e não exaustividade. É bem sabido que PIB e consumo de energia são altamente correlacionadas. Para estudos aprofundados sobre o assunto ver Stern (1993), Yang (2000), Soytas e Sari (2003), Shiu e Lam (2004), Oh e Lee (2004), Lee (2005), Lee e Chang (2007, 2008), Hu e Lin (2008), Sue Wing (2008). Por outro lado, o uso de PIB como output gera um efeito de manutenção do status quo, ou seja, dificulta a atribuição de maiores cotas de emissão a países pobres, o que pode impedir o seu desenvolvimento.

Quanto à não exaustividade, verifica-se que uma importante variável foi deixada de lado em 
Gomes e Lins (2008): a dimensão do país. Grandes emissões de $\mathrm{CO}_{2}$ em países de grande dimensão não são tão nocivas, já que ficam dispersas pela sua extensão geográfica. O inverso pode ser dito para países de pequena dimensão. Do ponto de vista teórico, há que se referir que a exaustividade desejada em problemas multicritério (ROY; BOUYSSOU, 1993) não é normalmente desejada em DEA. Isto porque o aumento de variáveis provocado pela busca da exaustividade aumenta a eficiência das DMUs, ou seja, nenhuma DMU é penalizada pela inclusão de novas variáveis. Como comentado na seção 2.3, em modelos DEA-GSZ com interesse na redistribuição e não na eficiência, a introdução de variáveis tanto pode melhorar quanto piorar o resultado de uma DMU.

Dessa forma, as variáveis escolhidas no modelo do presente artigo foram baseadas em Gomes e Lins (2008). Foram repetidas as variáveis que não apresentavam problemas de redundância, a saber, população, consumo de energia, (como outputs) e emissões de $\mathrm{CO}_{2}$ como output indesejável, tendo sido eliminada a variável PIB pela sua grande redundância com a variável consumo de energia. No seu lugar foi acrescentada a variável área, para medir a intensidade geográfica das emissões. As variáveis foram escolhidas por análise e opinião de pessoas envolvidas com o tema, sem o uso de métodos matemáticos de seleção de variáveis. Sobre o uso desses métodos deve ser ressaltado que Senra et al. (2007) indicam que a sua utilidade é limitada a certos casos especiais.

Em resumo, as variáveis do modelo para este artigo são: população $\left(10^{6}\right.$ habitantes), área $\left(10^{6} \mathrm{~km}^{2}\right)$, energia consumida $\left(10^{6} \mathrm{BTU}\right)$ como outputs e emissões de $\mathrm{CO}_{2}$ $\left(\mathrm{t}^{3}\right.$ de carbono equivalente) como output indesejável, aqui modelado como input. Os valores das variáveis população, emissão e consumo de energia são os usados por Gomes (2003), Gomes e Lins (2008); as áreas dos países foram obtidos de Ferreira (2003).

As DMUs do modelo DEA são as mesmas usadas por Gomes e Lins (2008): países signatários do Protocolo de Kyoto, incluindo-se aqueles chamados "países do Anexo I" (países desenvolvidos, responsáveis por $55 \%$ das emissões mundiais de $\mathrm{CO}_{2}$ ), para os quais havia informação disponível sobre todas as variáveis, em um total de 64 DMUs. Os dados usados são os da Tabela 1.

A escolha do modelo conduz a um dilema. O modelo BCC, em tese mais adequado devido às disparidades de escala entre os países, apresenta uma distorção grave ao tornar eficiente um país que tenha o maior valor em qualquer dos outputs, sem levar em conta os inputs (são as chamadas DMUs eficientes por default). No caso presente, China, Rússia e Estados Unidos seriam eficientes pela simples razão de terem, respectivamente, a maior população, a maior área e o maior PIB. Ora, estes países estão entre os maiores poluidores e ser eficiente seria aqui dar direito de emitir mais $\mathrm{CO}_{2}$, o que é um contrassenso.

$\mathrm{O}$ uso do modelo CCR apresenta um problema inverso. É extremamente rigoroso com países com pequenos valores das variáveis. Países pequenos, pouco populosos ou com baixo consumo de energia (indício de pobreza) ficariam como altamente ineficientes, o que os obrigaria a reduzir ainda mais as emissões de $\mathrm{CO}_{2}$, dificultando o seu desenvolvimento.

Assim, optou-se por um modelo DEA híbrido com retornos não decrescentes (ou crescentes) de escala (IRS), que se comporta com retornos variáveis para as DMUs de pequena dimensão e com retornos constantes para as DMUs de grande dimensão. Este modelo elimina os dois problemas mencionados anteriormente: dá chance aos pequenos países de aumentarem as emissões e não permite que isto seja feito pelos grandes poluidores.

\subsection{Resultados}

Com base neste conjunto de variáveis e com as medidas de eficiência geradas por DEA, foi calculado o modelo DEA-GSZ IRS para redistribuir as cotas de emissões de $\mathrm{CO}_{2}$ entre os países. Foi adotada a estratégia de redução proporcional, com todos os países ineficientes formando um único grupo de cooperação, conforme modelo teórico detalhado na seção 2. Com este modelo, procurou-se permitir que países mais eficientes pudessem poluir mais e privar países ineficientes de poluir, sem, no entanto, alterar o total emitido.

Como os softwares existentes não calculam o modelo DEA-GSZ e, a maioria não contempla os retornos híbridos de escala, o modelo foi calculado em duas etapas: primeiro rodaram-se os problemas de programação linear correspondentes ao DEA clássico com retornos híbridos de escala para cada DMU, usando-se o solver de uma planilha eletrônica. A aplicação da proporcionalidade referente ao modelo DEA-GSZ foi feita inserindo-se a Equação correspondente na mesma planilha.

Os resultados de eficiência são os da Tabela 2 e a realocação de cotas apresenta-se na Tabela 3.

\subsubsection{Modelo com valores originais de emissões}

Na Tabela 3, na coluna que mostra a variação em \%, verifica-se que é facultado a 41 dos 64 países analisados que aumentem suas emissões. Para os 23 demais é obrigatório que reduzam suas emissões.

Na Tabela 4, os países foram alocados em classes, segundo o percentual de aumento ou redução de emissões. É interessante observar que a maioria dos países pode aumentar sua poluição, sendo mantida constante a emissão total. Aqueles que devem diminuir 


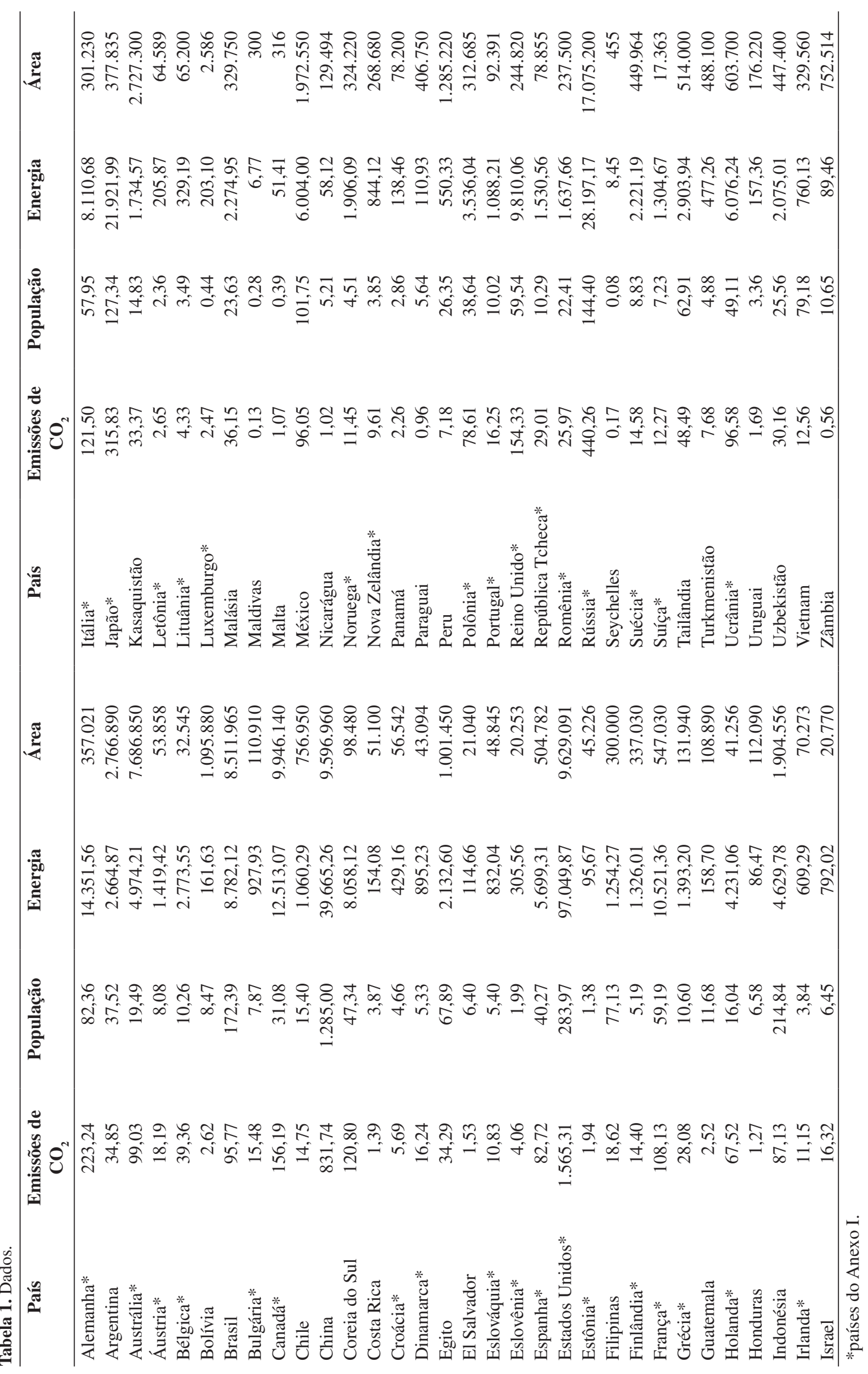




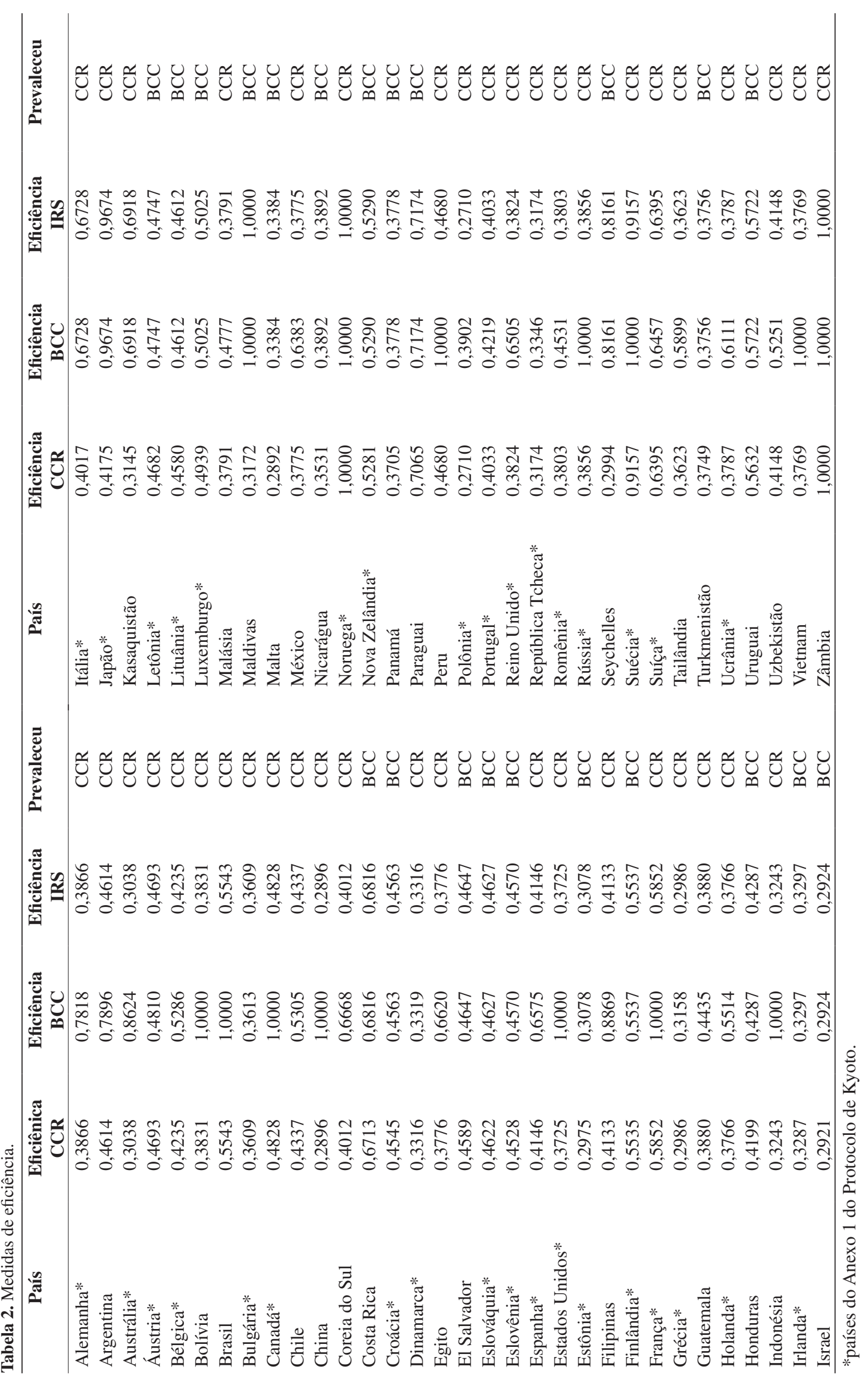




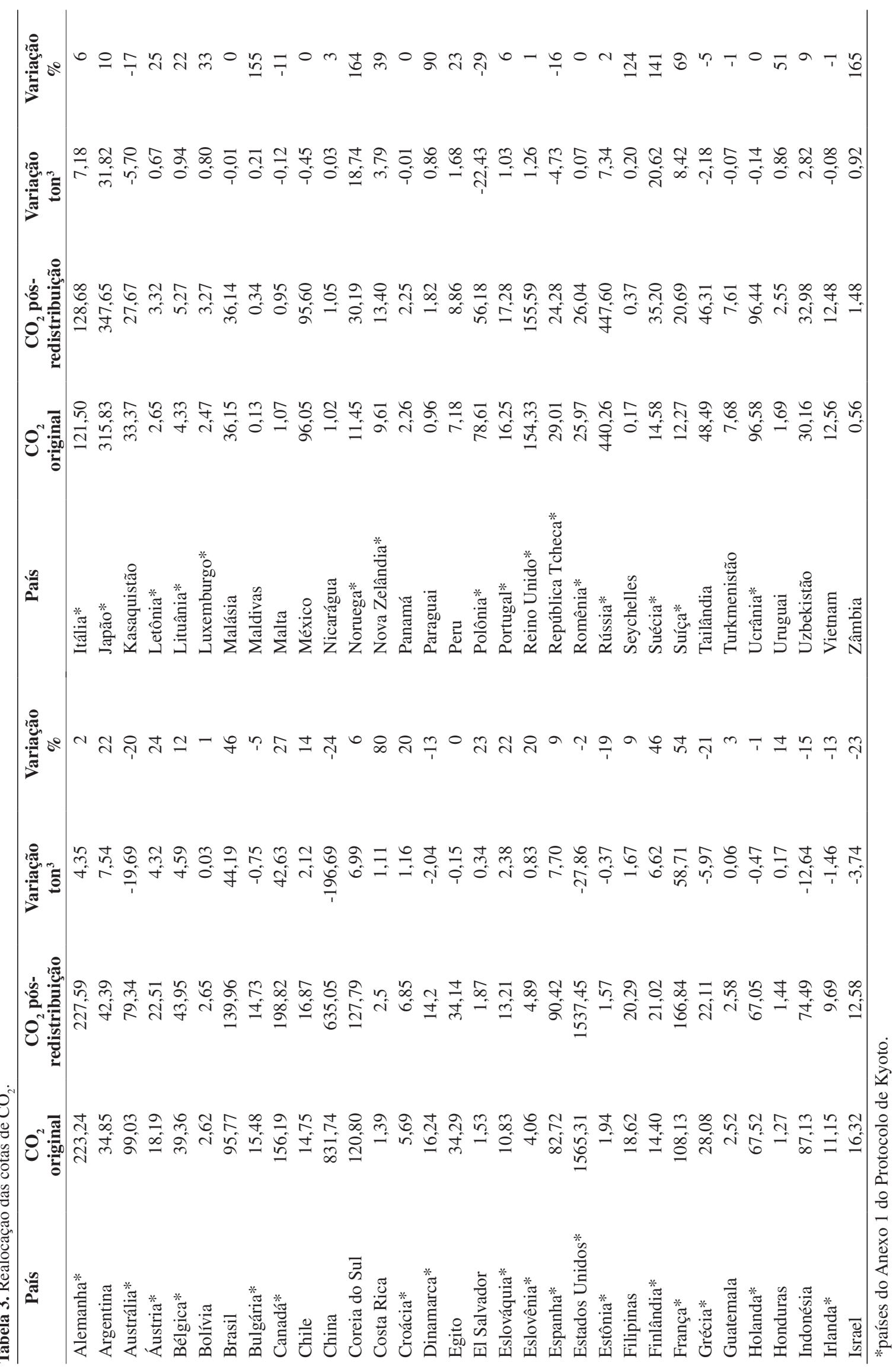


concentram-se no grupo de redução de até $20 \%$. Embora a alguns países seja permitido aumentar mais do que $50 \%$ de suas emissões, a nenhum dos países é solicitado que se reduza mais do que $50 \%$, o que seria bastante difícil de ser alcançado.

Conforme observado na Tabela 2, existem apenas três DMUs consideradas 100\% eficientes segundo a modelagem DEA IRS: Maldivas, Noruega e Zâmbia. A Noruega apresenta tal eficiência em função dos elevados níveis de energia consumida e a comparativa baixa emissão de $\mathrm{CO}_{2}$. Zâmbia é eficiente principalmente pelo baixo valor absoluto de poluição e razoáveis valores de extensão territorial. Maldivas possui o menor valor de emissões de $\mathrm{CO}_{2}$, e ao pertencer à região de retornos crescentes de escala está obrigatoriamente sobre a fronteira eficiente.

Ao realizar-se a mesma classificação da Tabela 4 apenas para os países do Anexo I, encontram-se os resultados da Tabela 5. Percebe-se que o grupo dos países do Anexo I possui um comportamento relativamente parecido com o grupo total. Este fato corrobora a ideia de que a questão da gestão das cotas de carbono não deve ficar restrita apenas a estes países. Não existe uma correlação direta entre o grupo ao qual o país pertence e a condição de bom poluidor e mal poluidor. A eficiência em poluir deveria ser o que efetivamente determinaria a gestão das cotas de emissões.

É interessante observar o "corte de eficiência" que determina se a DMU terá direito a aumentar a sua participação no mercado de emissões ou se ela terá que reduzi-la. No caso aqui em estudo, a fronteira está entre dois países, sendo um o de menor aumento, a Bolívia, que pode aumentar sua participação em apenas $0,026 \mathrm{t}^{3}$, e o Panamá, que deverá diminuir sua participação em $0,04 \mathrm{t}^{3}$. O corte acontece em aproximadamente $38 \%$ de eficiência, ou seja, países que possuam eficiência DEA IRS superior a este valor podem aumentar sua participação, enquanto que os países que possuem eficiência inferior devem reduzi-la.

Dentre os países que devem promover maiores reduções destacam-se China (29\% de eficiência) e Estados Unidos (37\% de eficiência). Conforme pode ser visto na Tabela 3, a China deveria reduzir suas emissões de dióxido de carbono em mais de
$196 \mathrm{t}^{3}$, o que corresponde a $24 \%$ de redução do total produzido. A China, apesar de ter valores elevados para as variáveis população, consumo de energia e extensão territorial, encontra-se na região de retornos constantes de escala do modelo IRS, dada a elevada emissão de $\mathrm{CO}_{2}$.

Já os Estados Unidos deveriam reduzir cerca de $28 \mathrm{t}^{3}$, o que não corresponde a mais de $2 \%$ do total emitido. Os Estados Unidos classificam-se de maneira semelhante à China, com elevados valores de população, consumo de energia e extensão territorial. Contudo, as emissões de $\mathrm{CO}_{2}$ não são tão excessivas comparativamente. Localizam-se também na região de retornos constantes, mas sua redução é menor do que aquela proposta para a China.

Um aspecto semelhante entre os dois países é que se a modelagem usada no problema fosse $\mathrm{BCC}$, por exemplo, ambos os países teriam direito a gerar mais emissões, pois possuem elevados valores em todas as variáveis. Seriam, dessa forma, beneficiados pela modelagem de retornos variáveis de escala.

Ainda é importante destacar que, embora por este modelo à China tenha sido imposta uma grande redução de suas emissões, segundo as regras do Protocolo de Kyoto não estaria obrigada a realizar tal redução, já que não faz parte dos países do Anexo I.

Entre os países aos quais foi facultado aumentar as emissões de $\mathrm{CO}_{2}$ encontram-se França e Brasil. Conforme pode ser visto na Tabela 3, a França estaria possibilitada a aumentar mais $58 \mathrm{t}^{3}$ de emissões, o que corresponde a $54 \%$ de aumento do total emitido. A França possui elevado consumo de energia e razoáveis valores de população e extensão territorial. Mesmo com elevada produção de $\mathrm{CO}_{2}$, encontra-se na região de retornos constantes, com valor de eficiência acima do valor de corte (59\%).

O Brasil poderia aumentar suas emissões em mais de $43 \mathrm{t}^{3}$, cerca de $46 \%$ de aumento do total produzido. Possui elevados valores de população e extensão territorial, mas razoável consumo de energia. É um moderado poluidor e também se encontra na região de retornos constantes, com eficiência acima do ponto de corte (55\%).

Tabela 4. Estratificação das DMUs, segundo os percentuais de alteração de emissões de $\mathrm{CO}_{2}$.

\begin{tabular}{lccccc}
\hline & Total de DMUs & Até 10\% & De 10 a 20\% & De 20 a 50\% & Mais que 50\% \\
\hline Aumentar & 41 & 13 & 4 & 14 & 10 \\
Diminuir & 23 & 11 & 8 & 4 & 0 \\
\hline
\end{tabular}

Tabela 5. Estratificação dos países do Anexo I, segundo os percentuais de alteração de emissões de $\mathrm{CO}_{2}$.

\begin{tabular}{lccccc}
\hline & Total de DMUs & Até $\mathbf{1 0 \%}$ & De 10\% a 20\% & De 20 a 50\% & Mais que 50\% \\
\hline Aumentar & 23 & 7 & 2 & 10 & 4 \\
Diminuir & 11 & 4 & 5 & 2 & 0 \\
\hline
\end{tabular}




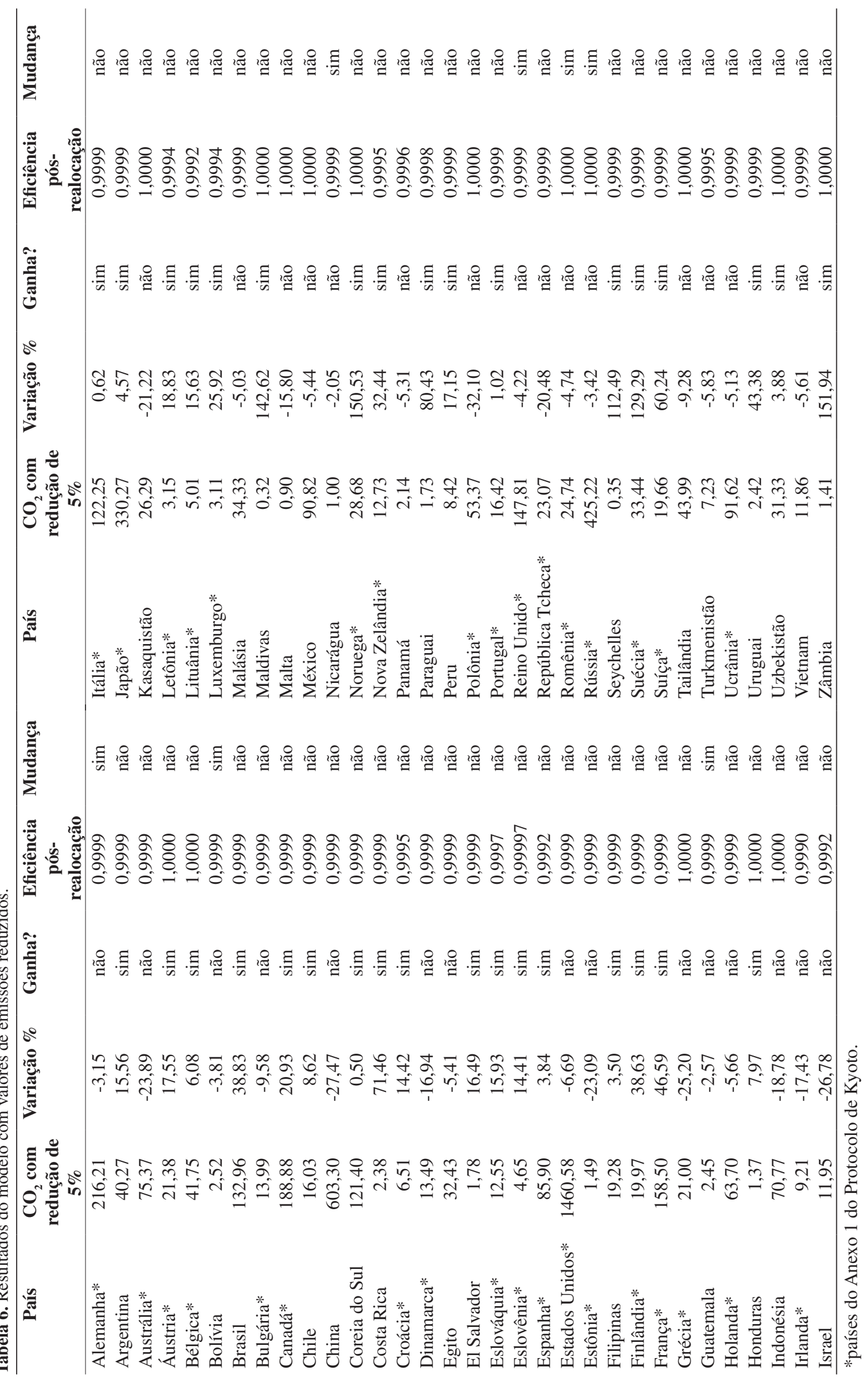




\subsubsection{Modelo com valores reduzidos de emissões}

No item anterior não foram impostas reduções no somatório total das emissões, conforme determinado pelo Protocolo de Kyoto. Entretanto, cabe recalcular a redistribuição de cotas que considere o percentual de redução de 5\% observado no Protocolo.

Como as medidas de eficiência DEA são calculadas de forma comparada e sendo a abordagem DEA-GSZ aqui usada modelada a partir da estratégia proporcional de alcance da fronteira, as DMUs guardam entre si uma relação de proporcionalidade. Isto garante que uma redução de 5\% no somatório total seja equivalente a uma redução de $5 \%$ em cada uma das cotas redistribuídas às DMUs. Ao se fazer isto, mantém-se ainda eficiência de $100 \%$ para todas.

Estes valores encontram-se na Tabela 6, coluna "Redução de 5\%", a qual equivale ao valor da coluna " $\mathrm{CO}_{2}$ pós-redistribuição" menos $5 \%$. É interessante observar que alguns países que antes, quando apenas houve a redistribuição, podiam ainda aumentar suas emissões, neste segundo cenário, que contempla a redução de 5\%, devem reduzi-las. Estes países estão identificados pelo "sim" da coluna "Mudança". Este é o caso, por exemplo, de Alemanha, Bolívia, Guatemala, Nicarágua, Reino Unido, Romênia e Rússia.

Deve-se ainda notar que o valor de corte da medida de eficiência, que separa os países entre aqueles que devem reduzir e os que podem aumentar as emissões, se desloca. Antes estava posicionado em $38 \%$ de eficiência entre o Panamá, que já estava obrigado a reduzir, e a Bolívia que possuía a menor cota de aumento. Neste segundo cenário, o valor de corte desloca-se para 40\%, aproximadamente. Os países que se encontram nesta fronteira são Nicarágua, que deve reduzir $0,03 \mathrm{t}^{3}$ de $\mathrm{CO}_{2}$, e Honduras, que pode aumentar suas emissões em $0,09 \mathrm{t}^{3}$.

\section{Conclusões}

O modelo DEA-GSZ apresenta uma inovação teórica bastante apropriada ao conceito de mecanismos flexíveis: o cenário referencial para a redistribuição de emissões, garantindo a máxima eficiência global. Aqui foi proposta a expansão dos modelos BCC e CCR para os casos de retornos crescentes e decrescentes de escala.

A obtenção de uma fronteira uniformizada, ou de máxima eficiência, mostra as possibilidades do modelo DEA-GSZ no apoio à decisão, em especial na sugestão de diretrizes às unidades produtivas para comportamentos futuros. Dessa forma, os resultados deste estudo de caso, no que se refere à redistribuição das cotas de emissão de $\mathrm{CO}_{2}$, podem representar uma referência para negociações baseadas em critérios técnicos e que reflitam uma justa alocação de responsabilidades, sem considerar aspectos puramente comerciais e/ou políticos.

A modelagem DEA-GSZ com retornos híbridos de escala mostrou-se adequada à gestão de cotas de emissões, obrigando que grandes países poluidores, como China e Estados Unidos, reduzissem os níveis de emissão de $\mathrm{CO}_{2}$. Por outro lado, esta abordagem também permitiu que países que possuíam pequenos valores de produção não fossem penalizados por tal. Estes resultados parecem ser mais coerentes do que os anteriormente encontrados por Gomes (2003) e Gomes e Lins (2008).

Aqui também não se impôs redução de emissões apenas aos países do Anexo I do Protocolo de Kyoto, assim como não se mostrou obrigatório que todos os países do Anexo I tivessem de reduzir suas emissões. Ao contrário, países pertencentes ou não ao Anexo I tiveram comportamento heterogêneo. Isto se insere de maneira coerente com os "mecanismos flexíveis" previstos no Protocolo de Kyoto. Adicionalmente, este modelo não impôs um corte linear das emissões, conforme estabelecido no Protocolo de Kyoto. Dessa forma, permite que os países realizem políticas particulares de desenvolvimento e de comportamento em relação aos seus níveis de emissão de $\mathrm{CO}_{2}$.

A inserção da variável área tornou o modelo mais rigoroso com países de alta densidade de poluição. Em um modelo DEA comum a inserção de uma variável sempre torna o modelo mais benevolente com as DMUs. No entanto, como foi usado um modelo com Ganhos de Soma Zero, a inclusão de uma variável adicional tornou o modelo mais rigoroso com as DMUs mal avaliadas nesta variável e benevolente com aquelas bem nela avaliadas.

\section{Referências}

AMIRTEIMOORI, A.; KORDROSTAMI, S.; SARPARAST,

M. Modeling undesirable factors in data envelopment analysis. Applied Mathematics and Computation, v. 180, n. 2, p. 444-452, 2006.

AVELLAR, J. V. G. Modelo de fronteira elipsoidal: um modelo paramétrico para a distribuição de inputs de soma constante com controle nos pesos. 2010. Tese (Doutorado em Engenharia Aeronáutica e Mecânica)Instituto Tecnológico de Aeronáutica, São José dos Campos, 2010.

AVELLAR, J. V. G.; MILIONI, A. Z.; RABELLO, T. N. Modelos DEA com variáveis limitadas ou soma constante. Pesquisa Operacional, v. 25, n. 1, p. 135-150, 2005.

AVELLAR, J. V. G.; MILIONI, A. Z.; RABELLO, T. N. Spherical frontier DEA model based on a constant sum of inputs. Journal of the Operational Research Society, v. 58, n. 9, p. 1246-1251, 2007.

BANKER, R. D.; CHARNES, A.; COOPER, W. W. Some models for estimating technical scale inefficiencies in Data Envelopment Analysis. Management Science, v. 30, n. 9, p. 1078-1092, 1984. 
CHARNES, A.; COOPER, W. W.; RHODES, E. Measuring the efficiency of decision-making units. European Journal of Operational Research, v. 2, p. 429-444, 1978.

COOPER, W. W.; SEIFORD, L. M.; TONE, K. Data envelopment analysis: a comprehensive text with models, applications, references and DEA-Solver software. USA: Kluwer Academic Publishers, 2000. 318 p.

FÄRE, R.; GROSSKOPF, S. Modeling undesirable factors in efficiency evaluation: comment. European Journal of Operational Research, v. 157, n. 1, p. 242-245, 2004.

FERREIRA, G. M. L. Atlas geográfico: espaço mundial. São Paulo: Moderna, 2003. 120 p.

GOMES, E. G. et al. Alocação de bolsas de iniciação científica às unidades da Embrapa com modelos de Análise Envoltória de Dados com Ganhos de Soma Zero. Engevista, v. 9, n. 1, p. 14-21, 2007.

GOMES, E. G. Modelos de análise de envoltória de dados com ganhos de soma zero. 2003. Tese (Doutorado em Engenharia de Produção)-COPPE/UFRJ, Rio de Janeiro, 2003.

GOMES, E. G.; AVELLAR, J. V. G. Modelos de análise de envoltória de dados com variáveis de soma constante: teoria e aplicação. Pesquisa Naval, v. 18, p. 99-104, 2005.

GOMES, E. G.; LINS, M. P. E. Modelling undesirable outputs with zero sum gains data envelopment analysis models. Journal of the Operational Research Society, v. 59, p. 616-623, 2008.

GOMES, E. G.; SOARES DE MELLO, J. C. C. B.; LINS, M. P. E. Busca seqüencial de alvos intermediários em modelos DEA com soma de outputs constante. Investigação Operacional, v. 23, n. 2, p. 163-178, 2003.

GOMES, E. G.; SOARES DE MELLO, J. C. C. B.; LINS, M. P. E. Redistribuição de inputs e outputs em modelos de análise envoltória de dados com ganhos de soma zero. Pesquisa Operacional, v. 24, n. 2, p. 269-284, 2004.

GOMES, E. G.; SOARES DE MELLO, J. C. C. B.; LINS, M. P. E. Uniformização da fronteira eficiente em modelos de análise de envoltória de dados com ganhos de soma zero e retornos constantes de escala. Pesquisa Operacional, v. 25, n. 2, p. 261-277, 2005.

GUEDES, E. C. C. Modelo de fronteira esférica ajustado: alocando input via DEA paramétrico. 2007. Dissertação (Mestrado em Engenharia Aeronáutica e Mecânica)Instituto Tecnológico de Aeronáutica, São José dos Campos, 2007.

HAILU, A.; VEEMAN, T. S. Non-parametric productivity analysis with undesirable outputs: An application to the Canadian pulp and paper industry. American Journal of Agricultural Economics, v. 83, n. 3, p. 605-616, 2001.

HU, J.-L.; LIN, C.-H. Disaggregated energy consumption and GDP in Taiwan: a threshold co-integration analysis. Energy Economics, 2008.

JAHANSHAHLOO, G. R. et al. Undesirable inputs and outputs in DEA models. Applied Mathematics and Computation, v. 169, n. 2, p. 917-925, 2005.

KUOSMANEN, T. Weak disposability in nonparametric production analysis with undesirable outputs. American Journal of Agricultural Economics, v. 87, n. 4, p. 1077-1082, 2005.

LEE, C.-C. Energy consumption and GDP in developing countries: a cointegrated panel analysis. Energy Economics, v. 27, n. 3, p. 415-427, 2005.

LEE, C.-C.; CHANG, C.-P. Energy consumption and economic growth in Asian economies: a more comprehensive analysis using panel data. Resource and Energy Economics, v. 30, n. 1, p. 50-65, 2008.

LEE, C.-C.; CHANG, C.-P. The impact of energy consumption on economic growth: evidence from linear and nonlinear models in Taiwan. Energy, v. 32, n. 12 , p. 2282-2294, 2007.
LINS, M. P. E. et al. Olympic ranking based on a Zero Sum Gains DEA model. European Journal of Operational Research, v. 148, n. 2, p. 312-322, 2003.

LOZANO, S. N.; VILLA, G. Centralized resource allocation using data envelopment analysis. Journal of Productivity Analysis, v. 22, p. 143-161, 2004.

MACEDO, M. A. Redistribuição das cotas de emissão de $\mathrm{CO}_{2}$ com modelos DEA-GSZ e retornos híbridos de escala. 2005. Dissertação (Mestrado em Engenharia de Produção)-Universidade Federal Fluminense, Niterói, 2005.

$\mathrm{OH}$, W.; LEE, K. Causal relationship between energy consumption and GDP revisited: the case of Korea 1970-1999. Energy Economics, v. 26, n. 1, p. 51-59, 2004.

RAMANATHAN, R. A multi-factor efficiency perspective to the relationships among world GDP, energy consumption and carbon dioxide emissions. Technological Forecasting and Social Change, v. 73, n. 5, p. 483-494, 2006.

RAMANATHAN, R. An analysis of energy consumption and carbon dioxide emissions in countries of the Middle East and North Africa. Energy, v. 30, n. 15, p. 2831-2842, 2005.

RAMANATHAN, R. Combining indicators of energy consumption and $\mathrm{CO}_{2}$ emissions: a cross-country comparison. International Journal of Global Energy Issues, v. 17, n. 3, p. 214-227, 2002.

RODRIGUES, J. A. P. et al. Estimating greenhouse gas emissions using computational intelligence. In: INTERNATIONAL CONFERENCE ON ENTERPRISE INFORMATION SYSTEMS, 11., Milan, Italy. Proceedings... 2009. p. 248-250.

ROY, B.; BOUYSSOU, D. Aide multicritère à la décision: méthodes et cas. Paris: Economica, 1993. 695 p.

SCHEEL, H. Undesirable outputs in efficiency valuations European Journal of Operational Research, v. 132, n. 2 , p. $400-410,2001$.

SEIFORD, L. M.; ZHU, J. Modelling undesirable outputs in efficiency evaluation. European Journal of Operational Research, v. 142, p. 16-20, 2002.

SENRA, L. F. A. D. C. et al. Estudo sobre métodos de seleção de variáveis em DEA. Pesquisa Operacional, v. 27, n. 2, p. 191-207, 2007.

SHIU, A.; LAM, P.-L. Electricity consumption and economic growth in China. Energy Policy, v. 32, n. 1, p. 47-54, 2004.

SOARES DE MELLO, J. C. C. B. et al. Algoritmo de alocação de recursos discretos com análise de envoltória de dados. Pesquisa Operacional, v. 26, n. 1, p. 225-240, 2006.

SOYTAS, U.; SARI, R. Energy consumption and GDP: causality relationship in G-7 countries and emerging market. Energy Economics, v. 25, n. 1, p. 33-37, 2003.

STERN, D. I. Energy and economic growth in the USA. a multivariate approach. Energy Economics, v. 15, n. 2, p. $137-150,1993$.

SUE WING, I. Explaining the declining energy intensity of the U.S. economy. Resource and Energy Economics, v. 30, n. 1, p. 21-49, 2008.

VENCHEH, A. H.; KAZEMI MATIN, R.; TAVASSOLI KAJANI, M. Undesirable factors in efficiency measurement. Applied Mathematics and Computation, v. 163, n. 2, p. 547-552, 2005.

VILLA, G.; LOZANO, S. A. Constant sum of outputs DEA model for Olympic Games target setting. In: INTERNATIONAL SYMPOSIUM ON DEA, 4., Aston University, US. Proceedings... 2004. p. 36.

YANG, H.-Y. A note on the causal relationship between energy and GDP in Taiwan. Energy Economics, v. 22, n. 3 , p. $309-317,2000$ 